\title{
Körperliche Leistungsfähigkeit regelmäßig trainierender, älterer Patienten im Vergleich zur Normalpopulation
}

\author{
Physical Performance of Older Patients Training Regularly: \\ Comparison with a Normal Population
}

Autoren

Institut
R. Ewert, A. Obst, O. Gürtler, B. Koch, S. Gläser, T. Bollmann

Universitätsmedizin Greifswald, Klinik für Innere Medizin B, Greifswald eingereicht 14.4.2014 akzeptiert nach Revision 19.5.2014

\section{Bibliografie}

Dol http://dx.doi.org/ 10.1055/s-0034-1377307

Online-Publikation: 8.7.2014

Pneumologie 2014; 68: 547-556

(c) Georg Thieme Verlag KG

Stuttgart $\cdot$ New York

ISSN 0934-8387

\section{Korrespondenzadresse}

Prof. Dr. med. Ralf Ewert

Ernst-Moritz-Arndt-Universität

Greifswald, Universitätsmedizin

Klinik für Innere Medizin B,

Bereich Pneumologie/

Infektiologie

F.-Sauerbruch-Straße

17475 Greifswald

ewert@uni-greifswald.de

\section{Zusammenfassung \\ $\nabla$}

Es wurde in den letzten Jahren mehrfach gezeigt, dass die kardiopulmonale Leistungsfähigkeit mit zunehmendem Alter sinkt. Auf der anderen Seite stellt die Leistungsfähigkeit im Sinne der Fitness einen wesentlichen Prognosefaktor dar. Somit wird verständlich, dass dem Erhalt bzw. der Wiederherstellung der Fitness im Rahmen der Rehabilitation ein großer Stellenwert beigemessen wird.

Wir haben 35 regelmäßig trainierende Probanden im Alter von 58-78 Jahren spiroergometrisch hinsichtlich ihrer Fitness untersucht und gegenüber gematchten Kontrollen aus einer Bevölkerungsstudie keine signifikanten Leistungsunterschiede nachweisen können. Zusätzlich haben wir die Sauerstoffkinetik mittels eines Stufentestes mit einer Last im aeroben Bereich bestimmt, um eine einfache Methode zur Erfassung der Fitness unter Alltagsbedingungen zu evaluieren. Überraschend zeigte die von uns gewählte Methode jedoch keine klinisch relevante Beziehung zwischen der Sauerstoffkinetik und der Fitness der Probanden.

\section{Einleitung}

$\nabla$

Es stellt ein allgemein anerkanntes Phänomen dar, dass die körperliche Leistungsfähigkeit mit zunehmendem Alter geringer wird. Dieses konnte jüngst wieder in der „Studie zur Gesundheit Erwachsener in Deutschland“ belegt werden [1]. In diese Studie wurden 8152 Personen zwischen 18 und 79 Jahren eingeladen, welche repräsentativ für die Wohnbevölkerung Deutschlands waren. Bei einer Teilpopulation von 2827 Personen im Alter von 18 - 64 Jahren konnte die kardiorespiratorische Fitness anhand eines submaximalen Belastungstestes auf dem Fahrradergometer gemessen werden. Ein wesentliches Ergebnis bestand darin, dass sich die Leistung pro Altersdekade

\section{Abstract \\ $\nabla$}

Previous studies indicate that physical performance, being a relevant prognostic parameter for cardiovascular events and mortality, decreases with age. Thus, the maintenance and restoration of physical performance as part of a rehabilitation program is of great interest.

In the present study, 35 physically active participants, aged 58-78 years, were investigated by cardiopulmonary exercise testing. In comparison to matched participants drawn from an epidemiological study, there was no significant difference in performance $g$ between the two groups. Additionally, oxygen kineticswere incrementally measured in order to evaluate a fairly simple procedure for determining fitness under activities of daily life. Surprisingly, this method did not reveal any clinically relevant association between oxygen kinetics and physical fitness.

um 4,2\% bei Männern und um 4,8\% bei Frauen reduzierte. In einer früheren australischen Untersuchung mit 1043 Personen im Alter von 18-78 Jahren konnte mit vergleichbarer Methodik ein etwa doppelt so großer Abfall der Leistung pro Altersdekade dokumentiert werden [2]. Anhand eigener Daten konnten wir in einer Bevölkerungsstichprobe „Study of Health in Pommerania“ (SHIP) auch einen Abfall der Leistung mit zunehmendem Alter bestätigen [3].

Dieses ist insofern bemerkenswert, da die kardiorespiratorische Leistungsfähigkeit seit langem als ein wesentlicher Prognosefaktor bekannt ist [46]. Dieser Sachverhalt wurde durch eine Studie mit 26000 Probanden im Alter von 18 - 83 Jahren während einer mittleren Beobachtungszeit von 
mehr als 10 Jahren nochmals bestätigt. In dieser Untersuchung wurde eine Assoziation zwischen kardiorespiratorischer Leistungsfähigkeit und dem Auftreten von kardiovaskulären Ereignissen aufgezeigt [7]. Folgerichtig geht eine erhöhte tägliche körperliche Aktivität (gemessen anhand des Energieverbrauchs) mit einer reduzierten Sterblichkeit einher, was bei 70-82-jährigen Personen nachgewiesen wurde [8].

Vor diesem Hintergrund wird verständlich, dass die bereits zu Zeiten Hippokrates' (460-377 v. C.) propagierte körperliche Ertüchtigung eine wesentliche Voraussetzung für das Erreichen eines hohen Alters darstellt. Dieses trifft umso mehr dann zu, wenn bereits kardiovaskuläre Risikofaktoren bzw. kardiorespiratorische Erkrankungen vorliegen [5]. Daher wird im Rahmen der Primär- bzw. Sekundärprävention sowie der Therapie chronischer Erkrankungen der langfristigen Rehabilitation ein hoher Stellenwert beigemessen. Beispielgebend ist die kardiologische Rehabilitation, deren primäres Ziel es ist, den Verlauf und die Prognose der zugrundeliegenden Erkrankung positiv zu beeinflussen. Als sekundäre Ziele werden die „Verbesserung der symptomfreien körperlichen Belastbarkeit, die Überwindung des entstandenen kardiovaskulären und muskuloskelettalen Funktionsverlustes, die psychologische Stabilisierung und soziale Wiedereingliederung sowie die positive Beeinflussung kardiovaskulärer Risikofaktoren“ genannt [9]. Für die Steuerung des Trainings im Rahmen der Rehabilitation, aber auch zur Bewertung der Ergebnisse der verschiedenen rehabilitativen Maßnahmen werden zunehmend spiroergometrische Parameter herangezogen (u.a. Spitzen-Sauerstoffaufnahme, $\mathrm{VO}_{2 \text { peak }}$ sowie die Sauerstoffaufnahme an der anaeroben Schwelle, $\left.\mathrm{VO}_{2} @ A T\right)$. Statt dieser globalen Messwerte kann auch die sog. „Sauerstoffkinetik“ zur Beschreibung der aeroben Fitness genutzt werden. Das lange bekannte theoretische Grundkonzept besteht darin, aus einer kurzen konstanten Belastung unterhalb der anaeroben Schwelle (hier folgt die Sauerstoffkinetik einer einfachen Exponentialfunktion [10, 11]) Aussagen zur Belastbarkeit bzw. der Veränderungen dieser nach unterschiedlichen Interventionen abzuleiten. Zur methodischen Umsetzung und klinischen Anwendung dieses Konzepts existiert eine Vielzahl von Studien. Bei gesunden Probanden [12 -15] wurden vorrangig methodische Untersuchungen durchgeführt und Normwerte [16] ermittelt.

Gegenstand unserer Untersuchung waren folgende Fragen: 1) Unterscheiden sich spiroergometrische Befunde der Probanden eines strukturierten Rehabilitationsprogrammes von denen gleichaltriger Normalpersonen? Und 2) Korreliert die Erfassung der „Sauerstoffkinetik“ zu Beginn der Belastung bei diesen Probanden mit Parametern der Sauerstoffaufnahme?

\section{Methodik}

$\nabla$

Probanden: Es wurden Patientinnen/-en aus einem seit Jahren etablierten ambulanten Rehabilitationsprogramm zur Teilnahme an der Untersuchung eingeladen. Unter dem Dach des „Rehasportvereins Greifswald e.V.“ führen die Patienten ein strukturiertes Kraft-Ausdauertraining an Geräten durch, wobei die Art und Intensität (Last/Wiederholungen) protokolliert wird. Weitere sportliche Aktivitäten im Sinne von Wandern bzw. alltäglichen Maßnahmen (z.B. Gartenarbeit, Radfahren) werden zusätzlich in Eigenregie durchgeführt. Die Charakterisierung der Probanden und der Trainingsintensität wird in der $\bullet$ Tab. 1 dargestellt. Die von uns in die Untersuchung eingeschlossenen Probanden waren 70 (Spanne 58 bis 78) Jahre alt und zeigen ein deutliches Über- wiegen des männlichen Geschlechts. Der Body Mass Index (BMI in $\mathrm{kg} / \mathrm{m}^{2}$ ) lag bei 25,3 . Über die Laborwerte konnte eine manifeste Anämie, eine gestörte renale Funktion bzw. in Kombination mit der klinischen Untersuchung eine manifeste Herzinsuffizienz bei allen Probanden ausgeschlossen werden.

Ein regelmäßiges Training wurde zum Zeitpunkt der Studie bereits über 36 Monate mit 2,5 Stunden/Woche durchgeführt. Bei den Indikationen zur Aufnahme des Trainings wurden kardiovaskuläre Erkrankungen, muskuloskelettale Störungen und altersbedingte Inaktivität („Alterstraining“) von den Probanden am häufigsten genannt. Der Charlson-Index [17] zeigte eine geringe Komorbidität der Probanden. Die Probanden waren Nichtraucher, wobei ein Proband (Nr. 18) seit 25 Jahren Ex-Raucher ist. Betablocker wurden lediglich von 13/35 Probanden eingenommen. Kontrollen: Die Kontrollgruppe wurde aus einer repräsentativen Bevölkerung aus Norddeutschland gewonnen [18] und erfolgte mit einem Matchingverhältnis von 1:4. Die Matching-Kriterien waren das Alter, das Geschlecht und die körperliche Fitness. Insgesamt wurden 130 Kontrollen ausgewählt.

Klinische und apparative Untersuchungen: Bei allen Probanden wurde eine Anamnese sowie eine klinische Untersuchung durchgeführt, wobei der Fokus auf der Erkennung von Kontraindikationen zur Durchführung der Spiroergometrie lag. Im Weiteren erfolgte eine komplette Lungenfunktionsdiagnostik inkl. Blutgasanalyse ( $\bullet$ Tab.2). Die lungenfunktionellen Befunde zeigten nur bei zwei Probanden eine obstruktive Ventilationsstörung $\left(\mathrm{FEV}_{1} /\right.$ FVC $<70 \%$ ), wobei in einem Fall eine chronisch obstruktive Lungenerkrankung bekannt war. In drei Fällen wurde eine restriktive Ventilationsstörung (VC bzw. TLC $<80 \%$ der Norm) dokumentiert. Die globale (DLCO $<80 \%$ der Norm) bzw. volumenkorrigierte (KCO <80\% der Norm) Diffusionskapazität war bei fünf bzw. zwei Probanden eingeschränkt.

Spiroergometrie: Nach schriftlicher Aufklärung und Einverständniserklärung wurden die Probanden auf einem elektromagnetisch gesteuerten Fahrradergometer in aufrecht sitzender Position (Ergoselect 200, Ergoline, Deutschland) entsprechend eines speziellen Protokolls (s.u.) untersucht. Gasaustausch und Ventilation wurden „breath by breath“ mit einer Gasanalyseeinheit (Innocor, Fa. Innovision, Odense, Dänemark) bestimmt. Die Registrierung des Zwölfkanal-EKGs und der Pulsoxymetrie erfolgten kontinuierlich, und alle 2 Minuten wurde der Blutdruck gemessen (Padsy-Ergo, Medset Medizintechnik GmbH).

Die spiroergometrische Untersuchung bestand aus zwei Abschnitten:

1. Zur Erfassung der „Sauerstoffkinetik“ wurde bei dem ruhig sitzenden Probanden eine 3-5-minütige Ruhephase mit Erreichen einer stabilen Sauerstoffaufnahme $\left(\mathrm{VO}_{2}\right)$ durchgeführt (Baseline 1). Dann wurde mit dem Treten ohne Last begonnen und nach fünf Minuten die $\mathrm{VO}_{2}$ ermittelt (Baseline 2). Anschließend folgte eine Belastung mit 25 Watt, und nach fünf Minuten wurde wieder die $\mathrm{VO}_{2}$ dokumentiert (Baseline 3).

2. Nach einer Ruhephase von fünf Minuten im Sitzen auf dem Fahrrad wurde eine symptomlimitierte Belastung nach einem modifizierten JONES Protokoll (20 Watt in der ersten Minute, dann minutenweise Steigerung um 16 Watt) durchgeführt [19]. Die Trittfrequenz konnte in beiden Abschnitten individuell gewählt werden, wobei der Wert von 50 Umdrehungen/ Minute nicht unterschritten wurde. Die Gasanalyse erfolgte bei der symptomlimitierten Belastung mit einer Mittelung von 30 Sekunden. 
这

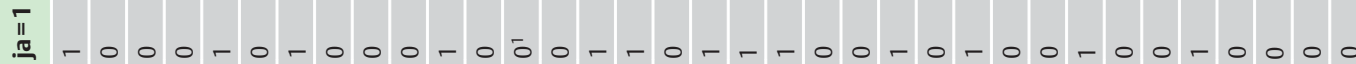

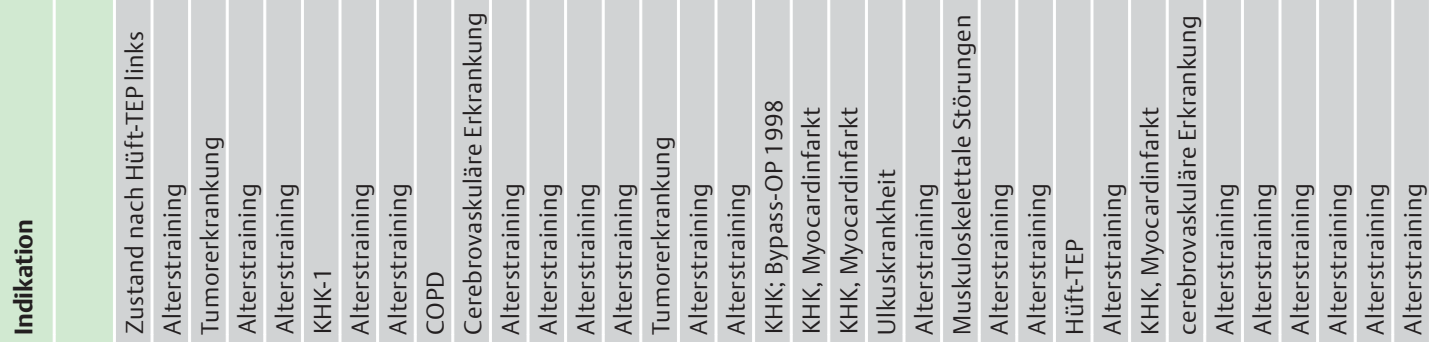

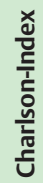

$0.0 n 00-00--0000 n-0 h--10000-0 m-000000$ 

胥

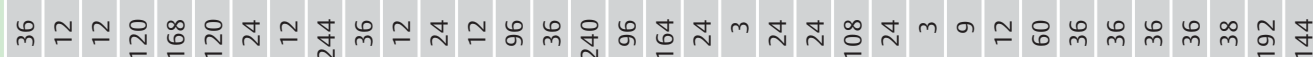

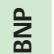

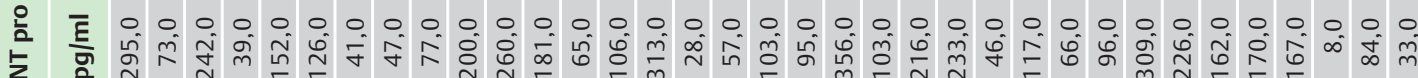
兹 产

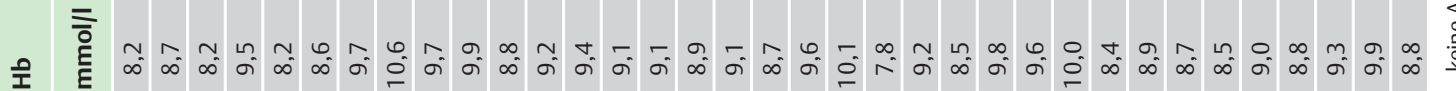

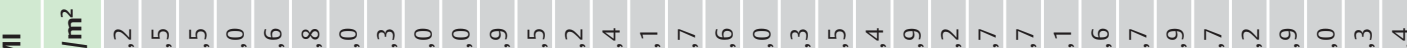

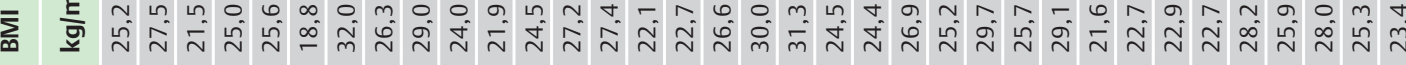
$\frac{\vec{n}}{3}$

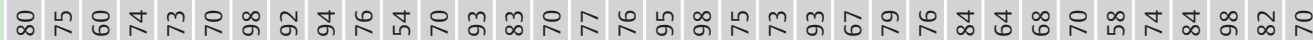
:

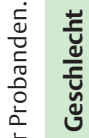
西

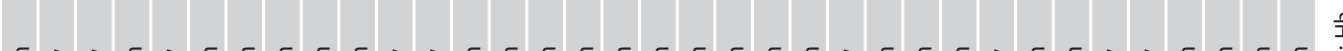
$\sqrt{\frac{\pi}{5}}$

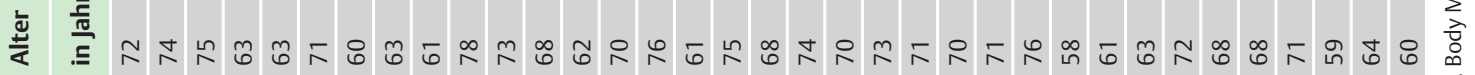




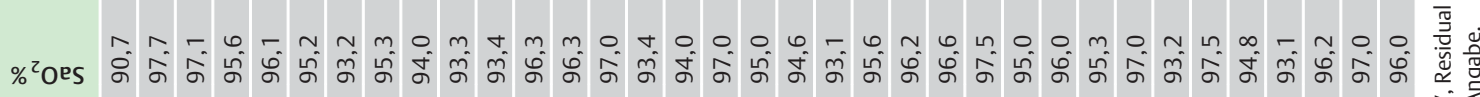

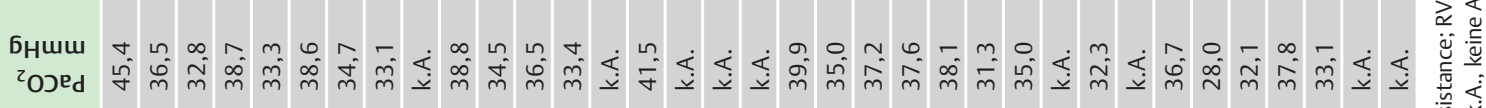

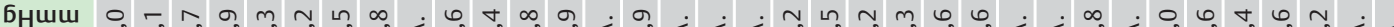
rord

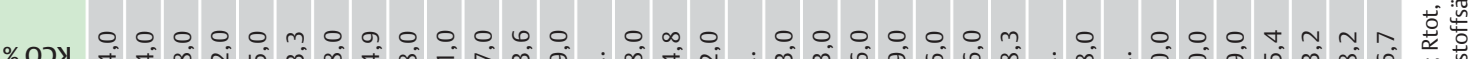

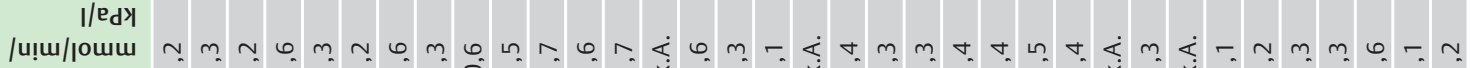

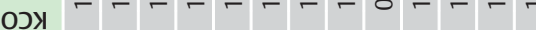
\% 0ग1

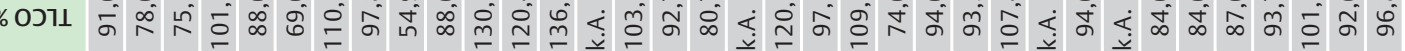

Bdy

|и!U/ןоum t

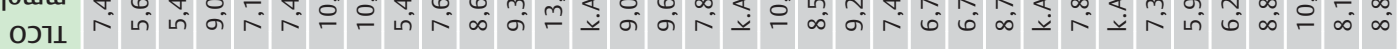

\%

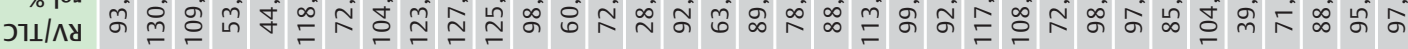

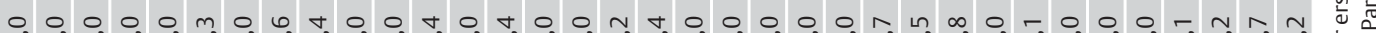

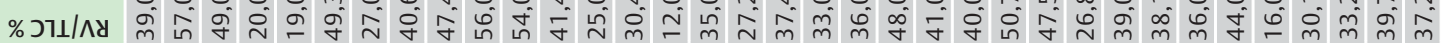
0 o 0 o 0 o 0 a 0 o m o r o \%/ヘy

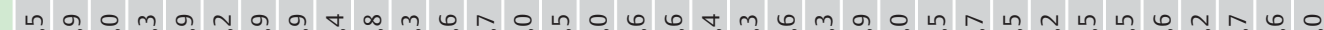

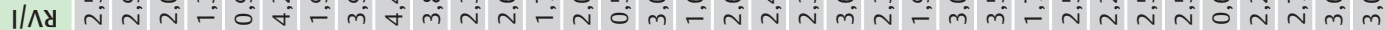

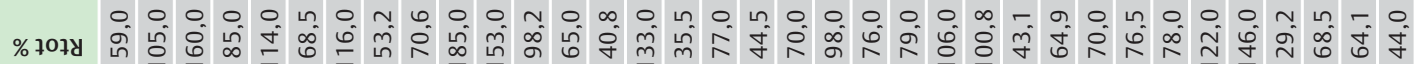

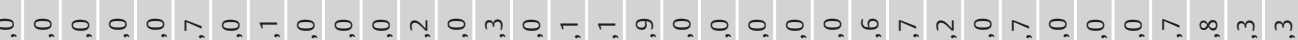
\%

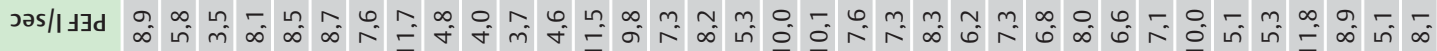

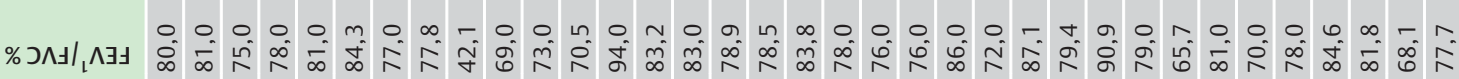

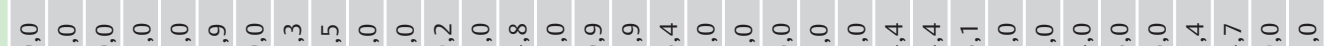

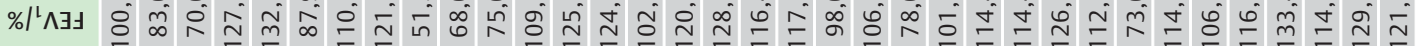

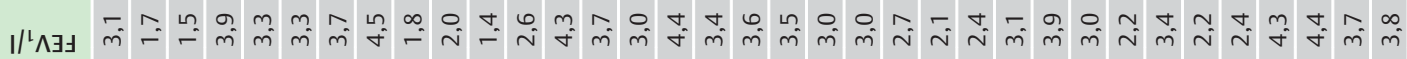

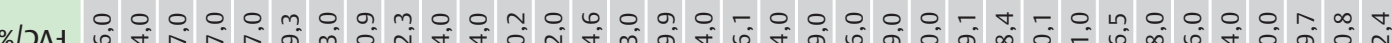
\%/गง

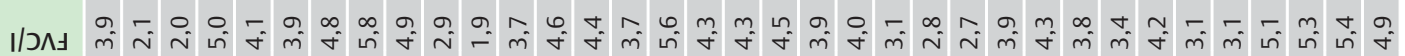

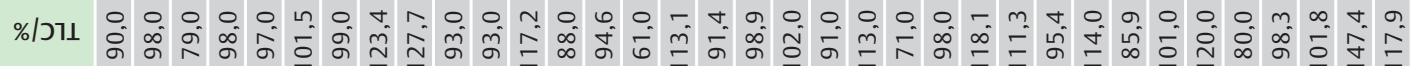

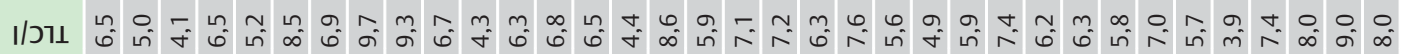
$\% / د \Lambda$ o $0.0 .0 .0 . m$. बं

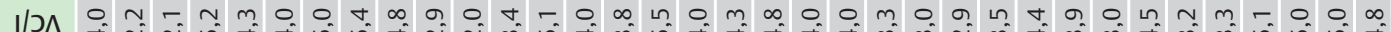

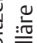
象 范 늘 焉 空 ¿

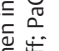

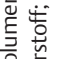
品 战违

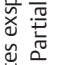
인 눈.

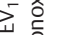




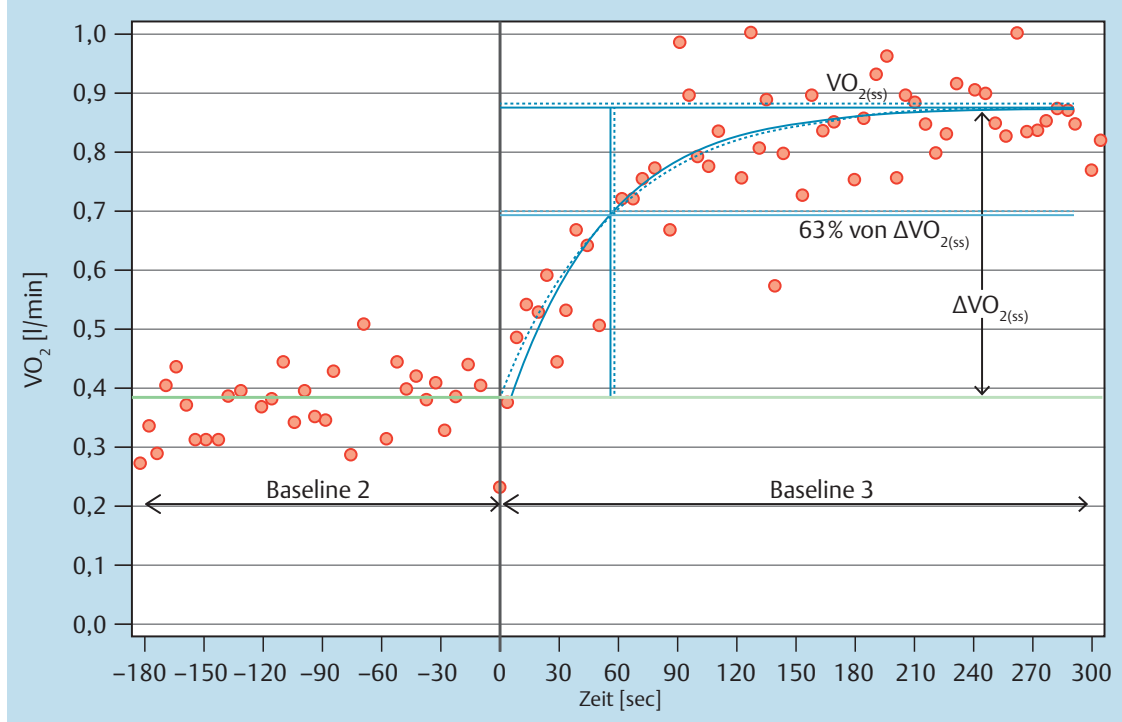

Abb. 1 Schematische Darstellung der Messung der Sauerstoffkinetik

Baseline 2: Treten ohne Last; Baseline 3: Belastungssteigerung auf 25 Watt; durchgehende Linie $\Delta \mathrm{VO}_{2(\mathrm{t})}=\Delta \mathrm{VO}_{2(\mathrm{ss})} \times\left(1-\mathrm{e}^{-(\mathrm{t}-\delta) / \mathrm{TVO} 2}\right) ;$ gepunktete Linie $\Delta \mathrm{VO}_{2(\mathrm{t})}=\Delta \mathrm{VO}_{2(\mathrm{ss})} \times\left(1-\mathrm{e}^{-(\mathrm{t}-\delta) / \mathrm{rVO}}\right)$, wobei $\delta=0$; $\Delta \mathrm{VO}_{2(\mathrm{ss})}$ ist die Differenzen der stabiler Sauerstoffaufnahme in der Ruhephase (Baseline 2) und der Sauerstoffaufnahme im steady-state in $\mathrm{ml} / \mathrm{min}$; $\delta$ ist die Zeitverzögerung; $\mathrm{r}$ ist die Zeitkonstante der Antwort der Sauerstoffaufnahme in Sekunden.

Zur Auswertung der Sauerstoffkinetik wurden die Rohdaten exportiert und mittels SAS 9.3 Software (SAS Institute Inc., Cary, NC) bearbeitet. Zur Bestimmung der Sauerstoffkinetik wählten wir den Übergang von der Baseline 2 zu 3, da hier keine „kardiopulmonale Phase der Adaptation“ berücksichtigt werden muss und somit die Sauerstoffkinetik einer einfachen Exponentialfunktion folgt ([10, 20]; 0 Abb. 1):

$\Delta \mathrm{VO}_{2(\mathrm{t})}=\Delta \mathrm{VO}_{2(\mathrm{ss})} \times\left(1-\mathrm{e}^{-(\mathrm{t}-\delta) / \tau}\right)$

$\Delta \mathrm{VO}_{2(\mathrm{ss})}$ Differenzen der stabilen Sauerstoffaufnahme in der Ruhephase (Baseline 2) und der Sauerstoffaufnahme im steadystate in $\mathrm{ml} / \mathrm{min}$,

$\delta$ ist die Zeitverzögerung (Delay).

$\tau$ ist die Zeitkonstante der Antwort der Sauerstoffaufnahme in Sekunden.

Die Regressionsanalyse erfolgte mittels nichtlinearer Kurvenanpassung der SAS Prozedur proc nlin. Die Berechnung der $\tau$-Werte erfolgte für zwei verschiedene $\mathrm{VO}_{2}$-Baseline (2)-Werte (Median ermittelt über 120 bzw. 180 Sekunden). Weiterhin wurden zwei Modelle für die benötigte Zeit, um $63 \%$ des Anstiegs der $\mathrm{VO}_{2}$ von der Ruhe (Baseline 2 ) bis zum Steady-State zu bewältigen ( $\triangleq$ TauWert), berechnet. Ist $\delta=0$ ist dieser Wert gleich $\tau$ und ist $\delta \neq 0$ ist dieser Wert $\delta+\tau$. Somit haben wir aus methodischer Sicht die Zeitkonstante $\tau$ im Rahmen der Anstiegskinetik der $\mathrm{VO}_{2}$ zu einer konstanten Last gewählt („on $\mathrm{TVO}_{2}$ “).

Die Auswertung der symptomlimitierten Belastung erfolgte mittels der Auswertesoftware der Gasanalyseeinheit, wobei die ventilatorische anaerobe Schwelle mit der V-slope-Methode ermittelt wurde [21,22]. Eine atemmechanische (ventilatorische) Limitierung wurde für zwei Konstellationen berechnet (MVV-1 als $\mathrm{FEV}_{1}$ x 35 bzw. MVV-2 als FEV $\mathrm{F}_{1}$ 41). Eine Limitierung wurde angenommen, wenn a) die maximale Ventilation mehr als $70 \%$ des berechneten Grenzwertes (MVV) bzw. b) mehr als 90\% des Grenzwertes erreichte. Die Darstellung der spiroergometrischen Werte erfolgte in Prozent der Norm nach den Wasserman/Hansen- [23] bzw. nach den SHIP-Normwerten [24].

Wiederholungsuntersuchungen: Bei 11 Probanden haben wir nach 21 Monaten eine nochmalige Untersuchung nach der o.g. Methodik vorgenommen, wobei die Sauerstoffkinetik bei einer Last von 50 Watt ermittelt wurde.

Datenerfassung/Statistik: Die Erfassung der Primärdaten erfolgte mittels Microsoft ${ }^{\circledR}$ Excel $^{\circledR}$ for Mac 2011 (Release 14.1.3). Alle Analysen wurden mit dem Statistikprogramm SAS 9.3 (SAS Institute
Inc., Cary, NC) durchgeführt. Sofern nicht anders ausgewiesen wurden alle Werte als Median dargestellt. Kontinuierliche Daten wurden als Median (1. Quartil; 3. Quartil) oder Mittelwert (Standardabweichung) angegeben. Nominale Daten wurden in Prozent angegeben. Für bivariate Analysen wurde der Mann-Whitney-UTest (kontinuierliche Daten) durchgeführt. Der Vergleich der CPET-Untersuchung zu zwei Zeitpunkten erfolgte mittels Wilcoxon-Rangsummentest. Ein p-Wert von $<0,05$ wurde als signifikant erachtet. Der Korrelationskoeffizient nach Spearman wurde angewendet, um mögliche Zusammenhänge der $\tau$-Werte mit den Messwerten der $\mathrm{VO}_{2} @ \mathrm{AT}$ bzw. $\mathrm{VO}_{2 \text { peak }}$ darzustellen.

Für die Untersuchung wurde ein positives Votum durch die Ethik-Kommission der Universitätsmedizin Greifswald (BB 103/ 11) erteilt.

\section{Ergebnisse}

Die kardiopulmonale Belastbarkeit der untersuchten Probanden wurde mit einer symptomlimitierten Belastung auf dem Fahrrad erfasst, wobei alle Probanden eine Ausbelastung (anhand eines RER > 1,1) erreichten ( Tab.3). Die maximale Leistung lag bei 91\% (Spanne 63-125) der Norm. Die Sauerstoffaufnahme an der anaeroben Schwelle (VO @AT) lag bei 95\% (Spanne 61 -141) und bei maximaler Belastung $\left(\mathrm{VO}_{2 \text { peak }}\right)$ bei $90 \%$ (Spanne $54-120$ ) der Norm. Für die Atemeffizienz (dargestellt an dem Wert VE/ $\mathrm{VCO}_{2} @ \mathrm{AT}$ ) lagen die Werte bei 104\% (Spanne 83-161) der Norm. Der endtidale Kohlendioxidpartialdruck an der anaeroben Schwelle (petCO $\mathrm{CAT}_{2}$ ) betrug 106\% (Spanne 87-127) der Norm. Der Sauerstoffpuls bei maximaler Belastung $\left(\mathrm{VO}_{2} / \mathrm{HR}\right)$ betrug 108\% (Spanne 61 -185) der Norm.

Bei Verwendung der weit verbreiteten Normwerte nach Wasserman/Hansen [23] lagen die Werte für die $\mathrm{VO}_{2 \text { peak }}$ bei $103 \%$ (Spanne 69-127) der Norm, und somit zeigten 30/35 Probanden eine normale kardiopulmonale Belastbarkeit (über $80 \%$ der Norm).

Die maximale Ventilation wurde mit 92\% (Spanne 58-119) der Norm erfasst. Eine atemmechanische (d.h. ventilatorische) Limitierung (VE max/MVV > 70\%) lag bei Verwendung des MVV-1 bei vier Probanden und für das MVV-2 bei zwei Probanden vor. Bei Verwendung des aktuell empfohlenen Grenzwertes von über 90\% zeigten zwei (MVV-1) bzw. kein Proband (MVV-2) eine atemmechanische Limitierung (Daten nicht dargestellt). 
Tab. 3 Spiroergometrische Befunde der Probanden.

\begin{tabular}{|c|c|c|c|c|c|c|c|c|c|c|c|c|c|c|c|}
\hline \multirow[t]{2}{*}{ Proband } & \multirow{2}{*}{$\begin{array}{l}\text { Leistung } \\
\text { maximal }\end{array}$} & \multirow{2}{*}{$\begin{array}{l}\text { Leistung } \\
\text { Norm \% }\end{array}$} & \multicolumn{10}{|c|}{ Ruhe } & \multicolumn{3}{|l|}{ AT } \\
\hline & & & HR & $\begin{array}{l}\text { syst. } \\
\text { BD }\end{array}$ & $\begin{array}{l}\text { diast. } \\
\text { BD }\end{array}$ & $\mathrm{VO}_{2}$ & $\mathrm{VE} / \mathrm{VCO}_{2}$ & $\operatorname{petCO}_{2}$ & $\mathrm{AF}$ & VE & $\begin{array}{l}\mathrm{O}_{2} l \\
\mathrm{HR}\end{array}$ & $\mathrm{SaO}_{2}$ & Last & $\mathrm{vo}_{2}$ & Norm \% \\
\hline 1 & 132 & 78,4 & 65 & 127 & 107 & 427 & 38,4 & 37,8 & 17 & 10,9 & 4,9 & 94 & 77 & 1118 & 94,4 \\
\hline 2 & 100 & 86,5 & 72 & 142 & 111 & 183 & 40,9 & 35,6 & 9 & 8,2 & 2,6 & 98 & 65 & 746 & 79,6 \\
\hline 3 & 100 & 88,3 & 74 & 120 & 89 & 188 & 45,3 & 40,0 & 12 & 8,3 & 2,5 & 98 & 66 & 679 & 78,2 \\
\hline 4 & 212 & 124,8 & 85 & 122 & 97 & 314 & 36,6 & 38,0 & 12 & 13,0 & 3,7 & 97 & 95 & 1206 & 103,1 \\
\hline 5 & 132 & 102,3 & 65 & 117 & 96 & 266 & 38,6 & 36,4 & 12 & 10,3 & 4,2 & 98 & 67 & 1033 & 108,2 \\
\hline 6 & 177 & 86,6 & 73 & 120 & 95 & 350 & 54,7 & 36,3 & 17 & 16,5 & 4,8 & 97 & 132 & 1698 & 141,4 \\
\hline 7 & 180 & 101,0 & 90 & 141 & 112 & 354 & 38,5 & 35,1 & 12 & 13,3 & 4,0 & 97 & 78 & 1439 & 108,3 \\
\hline 8 & 180 & 89,6 & 100 & 126 & 98 & 420 & 42,8 & 34,5 & 16 & 16,4 & 4,0 & 96 & 70 & 1290 & 96,7 \\
\hline 9 & 164 & 87,1 & 89 & 144 & 117 & 336 & 39,4 & 34,0 & 12 & 13,1 & 3,7 & k.A. & 68 & 1056 & 79,8 \\
\hline 10 & 100 & 62,7 & 75 & 128 & 98 & 230 & 57,8 & 33,8 & 16 & 11,8 & 2,5 & 97 & 84 & 1039 & 91,7 \\
\hline 11 & 84 & 84,8 & 70 & 128 & 93 & 193 & 57,7 & 30,5 & 21 & 12,0 & 2,7 & 97 & 47 & 670 & 81,8 \\
\hline 12 & 84 & 67,5 & 73 & 102 & 77 & 240 & 53,3 & 33,7 & 19 & 11,9 & 3,3 & 96 & 52 & 891 & 95,6 \\
\hline 13 & 210 & 106,1 & 73 & 117 & 86 & 334 & 50,1 & 34,7 & 18 & 13,0 & 4,7 & 96 & 84 & 1276 & 95,6 \\
\hline 14 & 196 & 120,8 & 69 & 127 & 94 & 290 & 49,0 & 36,4 & 17 & 12,9 & 4,4 & 97 & 99 & 1478 & 123,9 \\
\hline 15 & 132 & 80,8 & 68 & 131 & 105 & k.A. & k.A. & k.A. & 17 & 7,3 & k.A. & 96 & 84 & k.A. & k.A. \\
\hline 16 & 164 & 82,4 & 76 & 103 & 86 & 300 & 36,8 & 41,6 & 12 & 10,9 & 4,3 & 94 & 84 & 1147 & 91,8 \\
\hline 17 & 132 & 91,5 & 79 & 138 & 104 & 250 & 52,6 & 36,0 & 17 & 12,5 & 3,7 & 97 & 68 & 1054 & 95,1 \\
\hline 18 & 132 & 76,4 & 75 & 107 & 82 & 307 & 47,8 & 30,7 & 9 & 12,4 & 4,3 & 95 & 52 & 1031 & 80,2 \\
\hline 19 & 180 & 111,9 & 60 & 109 & 86 & 310 & 48,2 & 38,1 & 16 & 13,0 & 4,7 & 98 & 68 & 1086 & 85,7 \\
\hline 20 & 157 & 94,9 & 88 & 115 & 81 & 332 & 49,5 & 32,2 & 18 & 14,5 & 3,7 & 97 & 100 & 1117 & 96,8 \\
\hline 21 & 148 & 94,5 & 75 & 107 & 84 & 343 & 58,1 & 35,8 & 12 & 11,8 & 4,0 & 97 & 84 & 1210 & 108,1 \\
\hline 22 & 145 & 77,9 & 96 & 152 & 111 & 380 & 40,8 & 36,2 & 12 & 13,5 & 3,9 & 96 & 95 & 1499 & 115,8 \\
\hline 23 & 116 & 102,2 & 73 & 100 & 88 & 230 & 53,6 & 36,3 & 16 & 10,8 & 2,7 & 97 & 68 & 843 & 93,6 \\
\hline 24 & 94 & 82,0 & 96 & 154 & 113 & 400 & 30,7 & 27,5 & k.A. & 9,8 & 2,1 & 95 & 36 & 846 & 88,7 \\
\hline 25 & 164 & 109,8 & 81 & 137 & 100 & 262 & 45,9 & 35,7 & 14 & 10,9 & 3,3 & 98 & 74 & 1186 & 106,2 \\
\hline 26 & 148 & 86,0 & 70 & 141 & 114 & 354 & 40,4 & 31,8 & 22 & 11,7 & 4,7 & 97 & 68 & 1111 & 89,6 \\
\hline 27 & 125 & 94,5 & 86 & 114 & 96 & 255 & 38,9 & 35,8 & 12 & 10,3 & 3,0 & 96 & 65 & 835 & 90,6 \\
\hline 28 & 116 & 67,1 & 65 & 122 & 83 & 282 & 46,4 & 31,7 & 19 & 11,5 & 3,3 & 97 & 63 & 859 & 75,2 \\
\hline 29 & 158 & 97,0 & 67 & 122 & 100 & 263 & 51,2 & 32,4 & 14 & 12,3 & 4,0 & 98 & 90 & 1363 & 122,0 \\
\hline 30 & 100 & 92,3 & 77 & 142 & 107 & 230 & 39,4 & 34,2 & 13 & k.A. & k.A. & 96 & 53 & 791 & 92,7 \\
\hline 31 & 100 & 86,4 & 63 & 125 & 93 & 205 & 52,9 & 35,9 & 13 & 9,4 & 3,3 & 97 & 47 & 570 & 61,0 \\
\hline 32 & 164 & 94,3 & 63 & 120 & 96 & 265 & 45,3 & 37,1 & 13 & 12,1 & 4,7 & 97 & 100 & 1441 & 118,1 \\
\hline 33 & 244 & 117,3 & 71 & 136 & 100 & 320 & 51,6 & 33,9 & 16 & 14,3 & 3,6 & 96 & 100 & 1451 & 104,3 \\
\hline 34 & 196 & 105,9 & 71 & 120 & 96 & 295 & 44,3 & 34,9 & 7 & 10,4 & 4,1 & 97 & 106 & 1600 & 128,7 \\
\hline 35 & 164 & 92,5 & 76 & 118 & 89 & 248 & 47,9 & 35,4 & 8 & 11,4 & 4,0 & 96 & 90 & 1178 & 100,9 \\
\hline
\end{tabular}

Der Vergleich ausgewählter spiroergometrischer Parameter der Probanden mit den Kontrollen aus der Bevölkerungsstudie wird in der $\bigcirc$ Tab.4 dargestellt. Dabei wird für die Probanden ein signifikant höherer systolischer und diastolischer Blutdruck in Ruhe, petCO $\mathrm{CO}_{2} \mathrm{AT}$ und diastolischer Blutdruck bei maximaler Belastung nachgewiesen. Dagegen waren die maximale Ventilation, die maximale Atemfrequenz sowie die arterielle Sättigung bei $\mathrm{VO}_{2 \text { peak }}$ bei den Probanden signifikant geringer.

Somit kann zusammenfassend festgestellt werden, dass die kardiopulmonale Belastbarkeit (bestimmt über die erreichte Leistung und die sauerstoffassoziierten Parameter $\left[\mathrm{VO}_{2 \text { peak }}, \mathrm{VO}_{2} @ \mathrm{AT}\right.$ bzw. $\left.\mathrm{VO}_{2} / \mathrm{HR}\right]$ ) der Probanden keine signifikanten Unterschiede zur Kontrollgruppe auswiesen.

Mit der von uns gewählten Methodik der Erfassung der Sauerstoffkinetik konnten $\tau$-Werte nur für 28/35 Probanden berechnet werden ( $\bullet$ Tab.5). Die Daten zeigen, dass die Mittelung der Ausgangswerte für die $\mathrm{VO}_{2}$ über 120 bzw. $180 \mathrm{sec}$ (Baseline 2) keinen

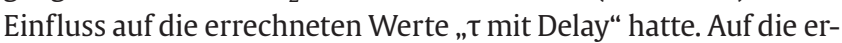

rechneten $\tau$-Werte hatte die gewählte Zeitmittelung jedoch bei einzelnen Probanden einen deutlichen Einfluss. Bei der Zweituntersuchung (andere Laststufe gewählt) waren 9/11 Untersuchungen auswertbar, und die errechneten $\tau$-Werte wurden kaum durch die unterschiedliche Zeitmittelung beeinflusst.

Es konnte bei keiner der beiden Zeitmittelungen eine signifikante Korrelation der $\tau$-Werte zu den Messwerten der $\mathrm{VO}_{2} @ A T(\tau$ mit Delay Korrelationskoeffizient nach Spearman 0,004 $(\mathrm{p}=0,985)$; $\tau 0,019(p=0,923)$ bei 120 sec Mittelung bzw. $\tau$ mit Delay 0,004 $(\mathrm{p}=0,985) ; \tau 0,085(\mathrm{p}=0,669)$ bei $180 \mathrm{sec}$ Mittelung oder $\mathrm{VO}_{2 \text { peak }}$ ( $\tau$ mit Delay $-0,182[p=0,335] ; \tau-0,141[p=0,475]$ bei $120 \mathrm{sec}$ Mittelung bzw. $\tau$ mit Delay $-0,182[p=0,335] ; \tau-0,053[p=0,789])$ nachgewiesen werden. 
Tab. 3 (Fortsetzung)

\begin{tabular}{|c|c|c|c|c|c|c|c|c|c|c|c|c|c|}
\hline & & & & Ende & & & & & & & & & \\
\hline $\mathrm{VE} / \mathrm{VCO}_{2}$ & Norm \% & petCO $_{2}$ & Norm \% & HR & $\begin{array}{l}\text { syst. } \\
\text { BD }\end{array}$ & diast. BD & $\mathrm{VO}_{2}$ & Norm \% & $\begin{array}{l}\text { Norm \% } \\
\text { (2) }\end{array}$ & $\mathrm{VE} / \mathrm{VCO}_{2}$ & $\operatorname{petCO}_{2}$ & $\begin{array}{l}\mathrm{O}_{2} \mathrm{l} \\
\mathrm{HR}\end{array}$ & Norm \% \\
\hline 26,2 & 91,6 & 50,0 & 126,6 & 84 & 138 & 91 & 1580 & 77,3 & 82,6 & 23,4 & 50,1 & 22,1 & 158,9 \\
\hline 32,2 & 120,5 & 45,0 & 115,0 & 108 & 214 & 98 & 1243 & 85,1 & 107,2 & 29,9 & 43,1 & 14,8 & 137,2 \\
\hline 26,5 & 101,2 & 42,5 & 107,0 & 146 & 188 & 118 & 734 & 54,4 & 69,1 & 25,7 & 43,6 & 12,2 & 121,4 \\
\hline 28,9 & 104,6 & 44,6 & 110,4 & 161 & 185 & 130 & 2290 & 108,1 & 112,5 & 24,8 & 39,4 & 14,3 & 104,5 \\
\hline 26,9 & 101,5 & 44,9 & 115,1 & 121 & 180 & 129 & 1645 & 103,4 & 120,6 & 24,9 & 45,3 & 13,5 & 124,1 \\
\hline 27,2 & 98,2 & 46,0 & 113,4 & 143 & 204 & 139 & 1850 & 81,1 & 94,1 & 25,8 & 41,4 & 12,9 & 91,0 \\
\hline 22,9 & 82,9 & 46,0 & 114,8 & 80 & 228 & 153 & 2425 & 103,5 & 104,3 & 32,3 & 38,5 & k.A. & k.A. \\
\hline 29,9 & 109,1 & 40,1 & 99,0 & 143 & 192 & 130 & 2538 & 103,3 & 105,6 & 27,5 & 42,9 & 17,8 & 112,0 \\
\hline 30,8 & 112,1 & 39,9 & 99,0 & 142 & 220 & 147 & 1955 & 81,8 & 82,9 & 30,9 & 40,4 & 13,7 & 87,4 \\
\hline 30,4 & 103,9 & 39,9 & 102,4 & 133 & 201 & 136 & 1295 & 68,5 & 76,5 & 28,4 & 40,4 & 17,0 & 128,3 \\
\hline 41,9 & 160,6 & 34,5 & 86,8 & 157 & 174 & 115 & 1073 & 87,9 & 103,9 & 37,3 & 33,3 & 6,8 & 74,1 \\
\hline 35,5 & 134,2 & 37,2 & 94,7 & 90 & 202 & 127 & 1305 & 86,0 & 104,2 & 31,9 & 38,6 & 14,8 & 138,2 \\
\hline 34,3 & 125,3 & 40,2 & 99,2 & 136 & 219 & 125 & 2345 & 95,8 & 97,4 & 28,3 & 42,8 & 24,2 & 152,0 \\
\hline 28,6 & 100,1 & 44,8 & 113,7 & 155 & 218 & 134 & 2446 & 120,3 & 126,6 & 24,7 & 43,7 & 15,8 & 112,9 \\
\hline k.A. & k.A. & 41,7 & 106,0 & 136 & 192 & 139 & 1569 & 82,2 & 93,2 & k.A. & k.A. & 12,3 & 95,1 \\
\hline 25,1 & 93,0 & 49,9 & 121,7 & 97 & 233 & 141 & 2138 & 89,7 & 94,4 & 24,5 & 47,0 & 22,9 & 155,4 \\
\hline 29,7 & 101,6 & 41,2 & 106,0 & k.A. & 223 & 152 & 1726 & 95,7 & 102,7 & 29,3 & 40,3 & 23,8 & 184,8 \\
\hline 30,4 & 107,0 & 37,0 & 93,8 & 138 & 191 & 116 & 1782 & 81,0 & 84,0 & 36,4 & 34,5 & 12,9 & 84,5 \\
\hline 27,8 & 95,1 & 43,1 & 111,4 & k.A. & 175 & 132 & 2436 & 118,2 & 125,0 & 24,8 & 48,0 & k.A. & k.A. \\
\hline 30,9 & 108,9 & 39,7 & 100,0 & 179 & 164 & 117 & 1442 & 71,6 & 76,6 & 33,4 & 41,6 & 8,2 & 61,0 \\
\hline 28,2 & 98,0 & 42,8 & 108,8 & 135 & 175 & 114 & 1915 & 100,6 & 109,1 & 31,8 & 38,4 & 14,1 & 108,5 \\
\hline 29,8 & 104,8 & 43,7 & 110,5 & 125 & 160 & 111 & 1994 & 88,2 & 93,3 & 30,3 & 42,6 & 15,9 & 103,5 \\
\hline 31,8 & 120,3 & 42,3 & 107,6 & 141 & 212 & 144 & 1225 & 86,6 & 104,0 & 27,9 & 40,4 & 8,6 & 83,5 \\
\hline 37,6 & 139,9 & 37,1 & 95,3 & k.A. & 206 & 112 & 1469 & 99,1 & 122,8 & 31,6 & 37,5 & k.A. & k.A. \\
\hline 29,8 & 101,9 & 38,5 & 98,9 & 95 & 271 & 166 & 2070 & 113,0 & 122,3 & 31,0 & 36,8 & 21,4 & 164,8 \\
\hline 34,3 & 125,6 & 37,9 & 93,4 & 137 & 193 & 127 & 1602 & 71,5 & 72,6 & 30,1 & 37,3 & 13,9 & 95,6 \\
\hline 27,3 & 104,4 & 44,3 & 112,5 & 132 & 170 & 111 & 1442 & 91,9 & 106,1 & 30,4 & 37,6 & 13,2 & 124,9 \\
\hline 35,1 & 127,7 & 39,2 & 96,6 & 118 & 174 & 103 & 1465 & 69,5 & 74,6 & 35,3 & 37,9 & 12,2 & 91,9 \\
\hline 29,8 & 104,5 & 36,4 & 91,9 & 150 & 212 & 130 & 2022 & 103,8 & 114,5 & 33,3 & 34,9 & 13,9 & 107,2 \\
\hline 29,6 & 113,2 & 45,0 & 113,6 & 150 & 207 & 137 & 1158 & 86,9 & 100,6 & 28,4 & 40,9 & 7,9 & 81,9 \\
\hline 32,5 & 121,8 & 39,7 & 101,8 & 128 & 210 & 144 & 1082 & 73,1 & 86,4 & 29,4 & 38,3 & 8,3 & 78,7 \\
\hline 26,8 & 94,1 & 45,5 & 115,1 & k.A. & 180 & 131 & 2017 & 95,2 & 101,0 & 29,4 & 39,5 & k.A. & k.A. \\
\hline 29,5 & 109,6 & 42,6 & 104,2 & 162 & 215 & 136 & 3110 & 119,8 & 119,5 & 27,1 & 35,6 & 19,2 & 115,5 \\
\hline 27,6 & 100,0 & 44,8 & 111,0 & 151 & 200 & 132 & 2454 & 108,2 & 111,7 & 27,9 & 43,6 & 16,6 & 113,0 \\
\hline 29,7 & 109,3 & 43,2 & 105,7 & 159 & 169 & 108 & 1968 & 90,0 & 94,9 & 30,1 & 39,0 & 12,1 & 89,2 \\
\hline
\end{tabular}

Abkürzungen: AT, Anaerobe Schwelle; HR, Herzfrequenz/Minute; syst. BD, systolischer Blutdruck in mmHg; diast. BD, diastolischer Blutdruck in mmHg; VO 2 , Sauerstoffaufnahme in $\mathrm{ml} / \mathrm{min}$; VE/ $\mathrm{VCO}_{2}$, Atemeffizienz als Quotient Ventilation/Kohlendioxidabgabe; petCO $\mathrm{CO}_{2}$, endtidaler Partialdruck für Kohlendioxid in mmHg; AF, Atemfrequenz/Minute; VE, Ventilation in I/min; $\mathrm{O}_{2} / \mathrm{HR}$, Sauerstoffaufnahme/Herzschlag in ml/Schlag; Norm [2] Normwerte nach Wasserman/Hansen [23]

\section{Diskussion}

In die Untersuchung wurden 35 Probanden in einem Alter zwischen 58-78 Jahren eingeschlossen, welche über längere Zeit im Rahmen einer „Alterssportgruppe“ ein regelmäßiges körperliches Training durchgeführt haben. Im Gegensatz zu anderen Studien unter Einbeziehung älterer Probanden $[3,25,26]$ haben wir Probanden mit kardiorespiratorischen Erkrankungen oder bestehendem Übergewicht (BMI >30) nicht ausgeschlossen. Somit wurde akzeptiert, dass Einflussfaktoren (sog. Confounder) auf ausgewählte spiroergometrische Parameter existieren. Hintergrund war die grundlegende Überlegung der Studie, einen Vergleich zwischen der sog. Normalbevölkerung (selektierte Kontrollen zur Erstellung von Normwerten) und regelmäßig trainierenden Probanden (mit/ohne Komorbiditäten) vorzunehmen. Über die gesamte Gruppe der Probanden gesehen, waren jedoch die aus den Voruntersuchungen [3] bekannten Confounder nur in geringem Umfang vorhanden. Diese Aussage wird durch die ge- ringen Auffälligkeiten bei den lungenfunktionellen Untersuchungen gestützt.

Zur Erfassung der kardiopulmonalen Belastbarkeit nutzten wir die symptomlimitierte Spiroergometrie auf dem Fahrrad. Die somit ermittelten Parameter der Leistung sowie verschiedene Werte der aeroben Kapazität $\left(\mathrm{VO}_{2 \text { peak}}, \mathrm{VO}_{2} @ \mathrm{AT}\right.$ sowie $\left.\mathrm{VO}_{2} / \mathrm{HR}\right)$ werden zunehmend als ein wichtiges Instrument der Trainingssteuerung angesehen $[9,27]$. Dieses gilt nicht nur für Patienten mit verschiedenen Erkrankungen, sondern auch für Empfehlungen zur körperlichen Belastung bei Normalpersonen (Breitensport). Bei unseren Probanden konnten wir sowohl für die erreichte Leistung in Watt (Fahrradergometer) als auch für alle Parameter der aeroben Kapazität keinen signifikanten Unterschied zur „Normpopulation" erfassen. Auch bei Verwendung der weit verbreiteten Normwerte nach Wasserman/Hansen [23] zeigten sich für den globalen Parameter der $\mathrm{VO}_{2 \text { peak }}$ keine signifikanten Unterschiede. Somit wurde die gute Vergleichbarkeit der im Rahmen von SHIP-1 generierten Werte zu den international weit verbrei- 
Tab.4 Vergleich ausgewählter spiroergometrischer Parameter zwischen Probanden und Kontrollen.

\begin{tabular}{|c|c|c|c|c|c|c|}
\hline Gruppe & Variable & Anzahl & $\mathrm{MW}( \pm \mathrm{SD})$ & Minimum & Maximum & $\mathbf{p}$ \\
\hline Probanden & Alter [Jahre] & 35 & $68( \pm 5,9)$ & 58 & 78 & \multirow{2}{*}{ n.s. } \\
\hline Kontrollen & & 130 & $68( \pm 5,6)$ & 58 & 78 & \\
\hline Probanden & \multirow{2}{*}{ Geschlecht (Frauen/Männer) } & $10 / 25$ & & & & \multirow{2}{*}{ n.s. } \\
\hline Kontrollen & & $37 / 93$ & & & & \\
\hline Probanden & \multirow[t]{2}{*}{ Größe $[\mathrm{cm}]$} & 35 & $174,3( \pm 8,3)$ & 157 & 193 & \multirow{2}{*}{0,004} \\
\hline Kontrollen & & 130 & $169,3( \pm 7,9)$ & 152 & 191 & \\
\hline Probanden & \multirow{2}{*}{ Gewicht [kg] } & 35 & $77,8( \pm 11,7)$ & 54 & 98 & \multirow{2}{*}{ n.s. } \\
\hline Kontrollen & & 130 & $81,2( \pm 13,9)$ & 49 & 125 & \\
\hline Probanden & \multirow[t]{2}{*}{ Maximale Leistung [Watt] } & 35 & $146,6( \pm 39,7)$ & 84 & 244 & \multirow{2}{*}{ n.s. } \\
\hline Kontrollen & & 130 & $145,4( \pm 42,6)$ & 68 & 260 & \\
\hline Probanden & \multirow[t]{2}{*}{ Belastungsdauer [sec] } & 35 & $546,3( \pm 149,1)$ & 320 & 860 & \multirow{2}{*}{ n.s. } \\
\hline Kontrollen & & 130 & $527,9( \pm 157,3)$ & 201 & 923 & \\
\hline Probanden & \multirow[t]{2}{*}{ Herzfrequenz (Ruhe) ( 1 /min) } & 35 & $75,7( \pm 10,0)$ & 60 & 100 & \multirow{2}{*}{ n.s. } \\
\hline Kontrollen & & 130 & $72,6( \pm 11,9)$ & 45 & 104 & \\
\hline Probanden & \multirow[t]{2}{*}{ Systolischer RR (Ruhe) (mmHg) } & 35 & $125,0( \pm 13,9)$ & 100 & 154 & \multirow{2}{*}{0,003} \\
\hline Kontrollen & & 130 & $116,3( \pm 18,1)$ & 73 & 179 & \\
\hline Probanden & \multirow[t]{2}{*}{ Diastolischer RR (Ruhe) (mmHg) } & 35 & $96,7( \pm 10,5)$ & 77 & 117 & \multirow{2}{*}{$<0,001$} \\
\hline Kontrollen & & 130 & $80,7( \pm 11,2)$ & 54 & 122 & \\
\hline Probanden & \multirow{2}{*}{ Sauerstoffaufnahme (Ruhe) (ml/min) } & 34 & $292,8( \pm 64,5)$ & 183 & 427 & \multirow{2}{*}{ n.s. } \\
\hline Kontrollen & & 130 & $305,6( \pm 69,0)$ & 100 & 450 & \\
\hline Probanden & \multirow[t]{2}{*}{$\mathrm{VO}_{2} @ \mathrm{AT}(\mathrm{ml} / \mathrm{min})$} & 34 & $1112,9( \pm 281,7)$ & 570 & 1698 & \multirow{2}{*}{ n.s. } \\
\hline Kontrollen & & 130 & $1025,5( \pm 273,1)$ & 500 & 2562 & \\
\hline Probanden & \multirow[t]{2}{*}{ VE/VCO $@$ AT } & 34 & $30,2( \pm 3,7)$ & 23 & 42 & \multirow{2}{*}{ n.s. } \\
\hline Kontrollen & & 130 & $29,0( \pm 3,8)$ & 22 & 45 & \\
\hline Probanden & petCO $@$ @AT $(\mathrm{mmHg})$ & 35 & $42,0( \pm 3,7)$ & 35 & 50 & $<0 \cap 01$ \\
\hline Kontrollen & & 130 & $37,4( \pm 5,4)$ & 23 & 49 & $<0,001$ \\
\hline Probanden & Herzfrequenz (maximal) (1/min) & 29 & $136,6( \pm 21,4)$ & 84 & 179 & \\
\hline Kontrollen & & 130 & $137,1( \pm 22,6)$ & 69 & 190 & n.s. \\
\hline Probanden & Systolischer RR (maximal) ( $\mathrm{mmHg}$ ) & 35 & $197,0( \pm 25,3)$ & 138 & 271 & \\
\hline Kontrollen & & 130 & $189,9( \pm 31,1)$ & 105 & 296 & n.s. \\
\hline Probanden & Diastolischer RR (maximal) (mmHg) & 35 & $127,9( \pm 16,4)$ & 91 & 166 & \\
\hline Kontrollen & & 130 & $91,7( \pm 13,9)$ & 39 & 117 & $<0,001$ \\
\hline Probanden & $\mathrm{VO}_{2}$ peak (ml/min) & 35 & $1794,6( \pm 520,4)$ & 1073 & 3110 & \\
\hline Kontrollen & & 130 & $1827,0( \pm 520,1)$ & 800 & 3045 & n.s. \\
\hline Probanden & $\mathrm{VE} / \mathrm{VCO}_{2}$ slope & 34 & $29,4( \pm 3,5)$ & 23 & 37 & $n s$ \\
\hline Kontrollen & & 130 & $29,0( \pm 3,7)$ & 21 & 45 & n.s. \\
\hline Probanden & Atemfrequenz (maximal) $(1 / \mathrm{min})$ & 35 & $25,7( \pm 4,6)$ & 18 & 41 & \\
\hline Kontrollen & & 130 & $30,9( \pm 6,2)$ & 16 & 47 & $<0,001$ \\
\hline Probanden & Ventilation (maximal) (L/min) & 34 & $59,8( \pm 13,8)$ & 38 & 94 & \\
\hline Kontrollen & & 130 & $68,6( \pm 21,2)$ & 30 & 120 & 0,023 \\
\hline Probanden & Sauerstoffpuls (maximal) (ml) & 31 & $14,8( \pm 4,6)$ & 7 & 24 & \\
\hline Kontrollen & & 130 & $13,7( \pm 3,3)$ & 6 & 23 & n.s. \\
\hline Probanden & Sättigung bei $\mathrm{VO}_{2}$ peak (\%) & 33 & $93,7( \pm 2,8)$ & 85 & 97 & \\
\hline Kontrollen & & 104 & $96,4( \pm 1,9)$ & 87 & 100 & $<0,001$ \\
\hline
\end{tabular}

Abkürzungen: siehe Tab. 3

teten Normwerten bestätigt. Ungeachtet des abweichenden Belastungsprotokolls konnte auch in einer jüngst publizierten Arbeit eine vergleichbare Leistungsfähigkeit bei 43 Probanden zwischen 60-79 Jahren dokumentiert werden. Es bleibt jedoch anzumerken, dass dieser Vergleich durch die Verwendung von Mittelwerten sowie geschlechts- und altersabhängige Darstellung der Ergebnisse erschwert ist. Die daraus resultierende geringe Anzahl der Probanden in den einzelnen Gruppen ist bei der Ableitung von Ergebnissen zu berücksichtigen. Dieses gilt auch für eine eigentlich sehr umfangreiche brasilianische Studie, die jedoch in der Altersgruppe 65-74 Jahre nur 10 Männer und 25 Frauen untersucht hat [25]. In SHIP-1 wurden dagegen in der Altersgruppe 65 - 74 Jahre noch 130 Frauen und 148 Männer und bei den über 75-Jährigen noch 23 Frauen und 51 Männer unter- sucht, und wir konnten über ein Matching von 1:4 insgesamt 130 Kontrollen für unseren Normwertvergleich auswählen.

Es existiert eine umfangreiche Literatur zur Bestimmung der sog. Sauerstoffkinetik, weshalb wir die Methode hinsichtlich der Praktikabilität unter Alltagsbedingungen bei unseren Probanden untersucht haben. Das eigentlich bestechende theoretische Grundkonzept besteht darin, über die Sauerstoffkinetik Aussagen zur kardiopulmonalen Belastbarkeit im Sinne der Fitness bzw. auch der Veränderungen nach Intervention (z. B. Training) abzuleiten. Bei Durchsicht der Literatur wird jedoch deutlich, dass methodische Details bei der Durchführung eine immense Bedeutung besitzen. So haben wir uns für die Erfassung über eine konstante Laststufe entschieden, welche gegenüber der Messung aus einem Stufen- bzw. Rampentest stabilere Werte erbringen soll 
Tab.5 Parameter der Sauerstoffkinetik.

\begin{tabular}{|c|c|c|c|c|}
\hline \multirow[t]{2}{*}{ Proband } & \multicolumn{2}{|c|}{ Mittelung $120 \mathrm{sec}$} & \multicolumn{2}{|c|}{ Mittelung $180 \mathrm{sec}$} \\
\hline & Tau+Delay & Tau & Tau+Delay & Tau \\
\hline 1 & 62 & 59 & 62 & 59 \\
\hline 3 & 29 & 26 & 29 & 20 \\
\hline 4 & 22 & 28 & 22 & 29 \\
\hline 5 & 49 & 50 & 49 & 54 \\
\hline 6 & 31 & 32 & 31 & 32 \\
\hline 7 & 48 & 45 & 48 & 45 \\
\hline 8 & 38 & 25 & 38 & 29 \\
\hline 9 & 43 & 54 & 43 & 55 \\
\hline 10 & 141 & 92 & 141 & 85 \\
\hline 12 & 77 & 38 & 77 & 38 \\
\hline 13 & 38 & 43 & 38 & 41 \\
\hline 14 & 62 & 55 & 62 & 60 \\
\hline 16 & 29 & 48 & 29 & 46 \\
\hline 17 & 60 & 74 & 60 & 72 \\
\hline 18 & 36 & 44 & 36 & 44 \\
\hline 21 & 60 & 71 & 60 & 86 \\
\hline 22 & 64 & 78 & 64 & 69 \\
\hline 23 & 46 & 47 & 46 & 40 \\
\hline 24 & 38 & 49 & 38 & 48 \\
\hline 25 & 69 & 58 & 69 & 58 \\
\hline 26 & 91 & 108 & 92 & 102 \\
\hline 27 & 40 & 40 & 40 & 39 \\
\hline 29 & 45 & 47 & 45 & 46 \\
\hline 30 & 55 & 59 & 55 & 56 \\
\hline 31 & 75 & 130 & 75 & 111 \\
\hline 32 & 61 & 72 & 61 & 68 \\
\hline 34 & 58 & 75 & 58 & 75 \\
\hline 35 & 113 & 84 & 113 & 86 \\
\hline \multicolumn{5}{|c|}{ Zweituntersuchung } \\
\hline 8 & 35 & 36 & 35 & 36 \\
\hline 10 & 51 & 74 & 51 & 74 \\
\hline 14 & 54 & 66 & 54 & 64 \\
\hline 17 & 50 & 50 & 50 & 48 \\
\hline 20 & 23 & 23 & 23 & 23 \\
\hline 21 & 50 & 58 & 50 & 48 \\
\hline 25 & 47 & 46 & 47 & 44 \\
\hline 32 & 48 & 54 & 48 & 54 \\
\hline 33 & 32 & 34 & 32 & 34 \\
\hline
\end{tabular}

[13]. Als weiteres haben wir uns für die Messung einer Zeitkonstanten ( $\tau$-Wert) aus einem „Steady State“ mit Treten ohne Last entschieden. Eine andere Möglichkeit wäre die Bestimmung der Kinetik aus der Ruhe auf eine konstante Last, welche als MRT (mean response time) auch klinische Anwendung gefunden hat. Mit diesem Wert wird die kardiopulmonale Phase der Belastung (Phase I) und $\mathrm{VO}_{2}$ Kinetik (Phase II) zusammen betrachtet. Bei der Wahl der Laststufen haben wir uns an der Literatur orientiert, wobei wir eine Last unterhalb der anaeroben Schwelle gewählt haben. Nur in diesem Bereich folgt die $\mathrm{VO}_{2}$ Kinetik einer monoexponentialen Funktion. Bei höheren Laststufen müssen andere Berechnungsmodelle genutzt werden [14,28,29]. Methodisch ist es zudem bedeutsam, ob man eine Anstiegs- (onset) oder Abfalls(recovery) Kinetik nutzt, wobei einzelne Daten auf eine bessere Reproduzierbarkeit der Abfallskinetik hinweisen [30]. In dieser Studie wurde auch der Einfluss der Mittelung ( 5 oder $10 \mathrm{sec} ; 5$ oder 8 Atemzüge) auf die MRT bzw. $\tau$-Werte untersucht, wobei die Mittelung über 5 Atemzüge die beste Reproduzierbarkeit erbrachte. Wir haben auch einen Einfluss der Zeitmittelung auf die $\tau$-Werte gefunden, wobei wir für unsere Studie deutlich längere Zeitmittelungen verwendet haben. Die Reproduzierbarkeit der gewonnenen Werte wird durch die mehrfache Untersuchung und nachfolgende Mittelung verbessert [15,31]. So wurden bei gesunden Probanden 6 Tests durchgeführt und gezeigt, dass die Mittelung über 4 Tests optimal ist [13].

Der von uns gewählte Ansatz zur Erfassung der $\mathrm{VO}_{2}$ Kinetik über die Zeitkonstante $\tau$ zu Beginn einer konstanten Laststufe kann für die Anwendung unter Alltagsbedingungen nicht empfohlen werden. Hintergrund dieser Aussage ist der hohe technische Aufwand (kompletter Datenexport und sekundäre Berechnung der Kinetik), die relevante Anzahl von Patienten ohne berechenbares Ergebnis und letztlich die fehlende Beziehung zu ausgewählten Parametern der Spiroergometrie. Ob die Ergebnisse durch eine Mehrfachmessung zu verbessern sind, muss offen bleiben. Jedoch würde auch die Notwendigkeit zur mehrfachen Durchführung dieses Testes die Anwendung in der klinischen Routine zur orientierenden Bestimmung der kardiopulmonalen Fitness nicht sinnvoll erscheinen lassen. Die bisherigen „Normwerte“ beziehen sich ausschließlich auf kleine Gruppen männlicher Probanden und weisen auf eine Altersabhängigkeit hin.

Durch die zunehmende Verfügbarkeit von Softwareanwendungen zur Bestimmung der $\mathrm{VO}_{2}$ Kinetik in den kommerziell verfügbaren Geräten erscheint eine breitere klinische Anwendung möglich. Es sei abschließend darauf verwiesen, dass im Gegensatz zu unseren Ergebnissen in der Literatur verschiedene Parameter der $\mathrm{VO}_{2}$ Kinetik erfolgreich zur Bewertung der Prognose und zur Dokumentation von Therapieeffekten bei verschiedenen Patientengruppen zum Einsatz kommen (u.a. Patienten mit Linksherzinsuffizienz, [32-36]).

\section{Interessenkonflikt}

Die Autoren geben an, dass kein Interessenkonflikt besteht.

\section{Literatur}

1 Finger JD, Krug S, Gößwald $A$ et al. Kardiorespiratorische Fitness bei Erwachsenen in Deutschland; Ergebnisse der Studie zur Gesundheit Erwachsener in Deutschland (DEGS1). Bundesgesundheitsbl 2013; 56: $772-778$

2 Gore CJ, Booth ML, Bauman A et al. Utility of pwc75\% as an estimate of aerobic power in epidemiological and population-based studies. Med Sci Sports Exerc 1999; 31: 348 - 351

3 Koch B, Schäper C, Ittermann T et al. Reference values for cardiopulmonary exercise testing in healthy volunteers: the SHIP study. Eur Respir J 2009; 33: 389-397

4 Ekelund LG, Haskell WL, Johnson JL et al. Physical fitness as a predictor of cardiovascular mortality in asymptomatic North American men. The Lipid Research Clinics Mortality Follow-up Study. N Engl J Med 1988; 319: $1379-1384$

5 Myers J, Prakash M, Froelicher $V$ et al. Exercise capacity and mortality among men referred for exercise testing. $N$ Engl J Med 2002; 346: $793-801$

6 Carnethon MR, Gulati M, Greenland P. Prevalence and cardiovascular disease correlates of low cardiorespiratory fitness in adolescents and adults. JAMA 2005; 294: 2981 - 2988

7 Sui X, LaMonte MJ, Blair SN. Cardiorespiratory fitness as a predictor of nonfatal cardiovascular events in asymptomatic women and men. Am J Epidemiol 2007; 165: 1413-1423

8 Manini TM, EverhartJE, Patel KV et al. Daily activity energy expenditure and mortality among older adults. JAMA 2006; 296: 171 -9

9 Bjarnason-Wehrens B (Hrsg.) Deutsche Leitlinie zur Rehabilitation von Patienten mit Herz-Kreislauferkrankungen (DLL-KardReha). Clin Res Cardiol 2007: (Suppl. 02): III/1-III/54

10 Whipp BJ. Rate constant for the kinetics of oxygen uptake during light exercise. J Appl Physiol 1971; 30: 261-263

11 Di Prampero PE, Davies CT, Cerretelli $P$ et al. An analysis of $\mathrm{O}^{2}$ debt contracted in submaximal exercise. J Appl Physiol 1970; 29: 547-51 
12 Bell C, Paterson DH, Kowalchuk JM et al. A comparison of modelling techniques used to characterise oxygen uptake kinetics during the on-transient of exercise. Exp Physiol 2001; 86: 667-676

13 Markovitz GH, Sayre JW, Storer TW et al. On issues of confidence in determining the time constant for oxygen uptake kinetics. Br J Sports Med 2004; 38: 553-560

14 Ozyener F, Rossiter HB, Ward SA et al. Influence of exercise intensity on the on- and off-transient kinetics of pulmonary oxygen uptake in humans. J Physiol 2001; 533: 891 - 902

15 Kilding AE, Challis NV, Winter EM et al. Characterisation, asymmetry and reproducibility of on- and off-transient pulmonary oxygen uptake kinetics in endurance-trained runners. Eur J Appl Physiol 2005; 93: $588-597$

16 Babcock MA, Paterson DH, Cunningham DA et al. Exercise on-transient gas exchange kinetics are slowed as a function of age. Med Sci Sports Exerc 1994; 26: 440-446

17 Charlson ME, Pompei P, Ales KL et al. A new method of classifying prognostic comorbidity in longitudinal studies: development and validation. J Chronic Dis 1987; 40: 373-383

18 Völzke H, Alte D, Schmidt CO et al. Cohort profile: the study of health in Pomerania. Int J Epidemiol 2011; 40: 294-307

19 Jones NL, Makrides L, Hitchcock C et al. Normal standards for an incremental progressive cycle ergometer test. Am Rev Respir Dis 1985; 131: 700-708

20 Mezzani A, Agostoni P, Cohen-Solal A et al. Standards for the use of cardiopulmonary exercise testing for the functional evaluation of cardiac patients: a report from the Exercise Physiology Section of the European Association for Cardiovascular Prevention and Rehabilitation. Eur J Cardiovasc Prev Rehabil 2009; 16: 249-267

21 Beaver WL, Wasserman K, Whipp BJ. A new method for detecting anaerobic threshold by gas exchange. J Appl Physiol (1985) 1986; 60: 2020-2027

22 Westhoff $M$, Rühle $\mathrm{KH}$, Greiwing A et al. Ventilatorische und metabolische (Laktat-)- Schwellen. Positionspapier der Arbeitsgemeinschaft Spiroergometrie. Dtsch Med Wochenschr 2013; 138: 275-280

23 Wasserman $K$ et al. Ed. Principles of exercise testing and interpretation: including pathophysiology and clinical applications. 5th Edition Philadelphia: Wolters Kluwer/Lippincott Williams \& Wilkins; 2012: $154-180$
24 Gläser S, Ittermann T, Schäper C et al. Referenzwerte für die Spiroergometrie - Ergebnisse der Study of Health in Pomerania (SHIP). Pneumologie 2013; 67: 58-63

25 Herdy $A H$, Uhlendorf $D$. Reference values for cardiopulmonary exercise testing for sedentary and active men and women. Arq Bras Cardiol 2011; 96: 54-59

26 Funk M, Schneider J. Spiroergometrische Referenzwerte für die sozialmedizinische Leistungsbeurteilung bei Erwachsenen im Alter über 60 Jahre. Pneumologie 2012; 66: 329-337

27 Bjarnason-Wehrens B (Hrsg.) Leitlinie körperliche Aktivität zur Sekundärprävention und Therapie kardiovaskulärer Erkrankungen. Clin Res Cardiol 2009: (Suppl. 04): 1-44

28 Scheuermann BW, Barstow TJ. $\mathrm{O}^{2}$ uptake kinetics during exercise at peak O2 uptake. J Appl Physiol 2003; 95: 2014-2022

29 Jones $A M$, Poole DC. Oxygen uptake dynamics: from muscle to mouthan introduction to the symposium. Med Sci Sports Exerc 2005; 37: $1542-1550$

30 Kemps HM, De Vries WR, Hoogeveen AR et al. Reproducibility of onset and recovery oxygen uptake kinetics in moderately impaired patients with chronic heart failure. Eur J Appl Physiol 2007; 100: 45-52

31 Zeiselmeier A. Die Kinetik der Sauerstoffaufnahme bei Belastungsbeginn in Relation zum Herzfrequenzanstieg und der alveolären Sauerstoffkonzentration. Dissertation Tübingen: 2006

32 Schalcher C, Rickli $H$, Brehm M et al. Prolonged oxygen uptake kinetics during low-intensity exercise are related to poor prognosis in patients with mild-to-moderate congestive heart failure. Chest 2003; 124: $580-586$

33 Sietsema KE, Ben-Dov I, Zhang YY et al. Dynamics of oxygen uptake for submaximal exercise and recovery in patients with chronic heart failure. Chest 1994; 105: $1693-1700$

34 Koike A, Koyama $Y$, Itoh $\mathrm{H}$ et al. Prognostic significance of cardiopulmonary exercise testing for 10 -year survival in patients with mild to moderate heart failure. Jpn Circ J 2000; 64: 915-920

35 Brunner-La Rocca HP, Weilenmann D, Follath $F$ et al. Oxygen uptake kinetics during low level exercise in patients with heart failure: relation to neurohormones, peak oxygen consumption, and clinical findings. Heart 1999; 81: $121-127$

36 Arena R, Humphrey R, Peberdy MA. Measurement of oxygen consumption on-kinetics during exercise: implications for patients with heart failure. J Card Fail 2001; 7: $302-310$ 